\title{
Why a Focus on Complementary Treatments?
}

\author{
Raimund Jakesz Barbara Jakesz \\ Klinische Abteilung für Allgemeinchirurgie, Medizinische Universitätsklinik Wien, Austria
}

Since the initiation of BREAST CARE, it has been my prime objective as one of the Editors-in-Chief, to address relevant issues or invite others to do so, in order to provide information on the most recent developments and research results, while also presenting future prospects. Selecting the key topic of this issue, we face the need to address the 'significance of complementary medicine', and also to set this 'hot potato' into relationship with the journal's approach to adhere to scientific standards. In this field the 'public opinion', the 'needs of those concerned', and the 'power of facts' particularly diverge from the views of modern, evidence-based medicine.

We, as physicians, increasingly see ourselves confronted with our patients' and their relatives' specific questions: 'What is the reason for my disease? Is there a deeper meaning? Could complementary treatments heal me or bring me relief?'

The needs of the patients entrusted to us have in may ways experienced strong change in the course of the past decades, the media and the internet now playing a key role in opinionmaking and the dissemination of information. We are thus brought to recognize the fact that our 'market' has clearly outlined requirements - just to mention a few:

- sensitive consideration and understanding for the exigencies and questions of those concerned;

- explanations for the causes of malignant diseases;

- involvement of the patients in the process of recovery;

- psychic and physical quality-of-life improvements in the course of treatment;

- support in improving the overall prognosis by altering life perspectives and habits in multiple ways, particularly with respect to the importance attached to one's own inner healing.

Topics such as healthy nutrition, spirituality, emotional wellbeing and the body-soul-spirit relationship are omnipresent in the media and public considerations. In many cases, this circumstance may lead to patients feeling rejected and not un- derstood, questioning their confidence in the medical profession, and potentially making adverse decisions in such situations. Complementary approaches have gained such significance in the public that we feel the need, for the benefit of our patients, to be at least basically informed about the various approaches and their efficacy. This is the single way to ensure that we may support our patients with compassion, respect and sympathy.

Answers frequently given to the question, 'How do you see the difficulty of guiding cancer patients and instructing them to deal with their disease?' include the following: 'I haven't been trained and don't have the time to deal with such things in my daily practice.' 'I don't want to engage in the emotional level.' 'I don't know how to handle situations when cancer patients address their distress.' 'I believe complementary medicine is a matter of pure profiteering and charlatanism.' 'My personal convictions with regard to spirituality and emotional problem-solving have nothing to do with my professional life as a physician.' 'I might know how to give my patients an understanding of these matters, however, I'm not confident enough to address emotional or spiritual issues.'

Now, where does this attitude come from? Thousands of years ago, in highly civilized cultures and their ancient traditions, the holistic perspective of the body, mind and soul - and disease as a disharmony in these constituents' interplay - was state-ofthe-art, and thus a matter of course. This holistic approach not only applied to patient management, but also to the therapist who was a physician, healer, priest and philosopher all in one. In general, the spiritual component always was integral to physical healing.

The holistic perspective experienced a change as of the 17th century, partly due to René Descartes who subjected the human body to a 'mechanistic' model, thus initiating a process in which medicine was to become a purely natural science and rejecting its humanistic aspect. As a final consequence, this

\begin{tabular}{|c|c|}
\hline KARGER & (c) 2007 S. Karger GmbH, Freiburg \\
\hline $\begin{array}{l}\text { Fax +49 } 7614520714 \\
\text { E-mail Information@Karger.de } \\
\text { www.karger.com }\end{array}$ & $\begin{array}{l}\text { Accessible online at: } \\
\text { www.karger.com/brc }\end{array}$ \\
\hline
\end{tabular}

Univ. Prof. Dr. Dr. h.c. Raimund Jakesz Medizinische Universitätsklinik Wien

Klinische Abteilung für Allgemeinchirurgie

Währinger Gürtel 18-20, 1090 Wien, Austria

Tel. +43 1 40400-6916 Fax -6918

E-mail raimund.jakesz@meduniwien.ac.at 
process resulted in evidence-based medicine superbly translating, on a physical level, the results of large studies into guidelines and directives. Thus, the quality of physically related treatments went through enormous progress. Patients' diagnostics and therapies are now comprehensibly ascertained in the framework of clear standards.

In our perspective, it is necessary to additionally consider humanistic aspects in an attempt, on the foundations of evidence-based medicine, to deal with the importance of nonphysical processes in the induction of diseases, dealing with the diseases themselves, the process of healing and the process of maintaining health. We are not only physical beings, we also have emotions and thoughts that impact the physical body, as is sufficiently well known from and proven within psychosomatic medicine. Consequently, we as physicians act out our true potential only in part if we limit ourselves to the purely physical component and negate the mental-emotional-spiritual field in which we have not been primarily trained. We feel this development holds several hazards: Our treatments do not embrace human entireties but rather only parts, the visionary development of medicine is barely educible if limited to the physical level, we insufficiently satisfy our patients' re- quirements and needs, as mentioned above, and we have handed some of the competencies we are primarily entitled to over to other professions that are developing on a parallel and independent level, without our information and influence.

Evidence-based medicine, postulated as our maxim of action, is doubtlessly in an ambivalent relationship with complementary medicine, as the academic soundness in the latter field has by far not reached the scientific level of traditional Western medicine. Simply, large clinical studies exploring the meaning of emotions and emotional management with respect to specific spiritual issues are incomparably more difficult to perform.

Therefore, we should like to appeal for and encourage an enhanced spectrum of medical performance, particularly in oncology. We believe there indeed is a potential and that markedly increasing efforts are necessary not only to alleviate the needs of those concerned, but also to perceive connections, develop novel treatment strategies and let medicine again become a holistic science.

Every one of us has a body, feelings, a mind and a soul, and all these aspects are reflected in the significance of disease and health. 